\title{
RENDIMIENTO DE Stevia rebaudiana Bert. BAJO TRES ARREGLOS POBLACIONALES EN EL SINÚ MEDIO
}

\section{YIELD OF Stevia rebaudiana Bert. UNDER THREE POPULATION ARRANGEMENT IN MIDDLE SINU}

\author{
Miguel Espitia C. ${ }^{1}$ \\ Rafael Montoya B. ${ }^{2}$ \\ Liliana Atencio S. ${ }^{3}$
}

\section{RESUMEN}

El estudio tuvo como objetivo evaluar el efecto de tres arreglos de población sobre el rendimiento de hojas en dos genotipos de Stevia rebaudina Bert., bajo las condiciones del Sinú Medio (Montería - Colombia), durante 16 semanas, del primer semestre de 2006. Se evaluaron seis tratamientos en arreglo factorial $(2 \times 3)$, originados de la combinación de dos genotipos de estevia (Morita 1 y Morita 2) y tres arreglos de siembra (20x20; 40x20 y $40 \times 40 \mathrm{~cm}$ entre surcos y plantas, respectivamente); para ello, se utilizó un diseño de bloques completos al azar con cuatro repeticiones. Se evaluaron las variables: rendimiento de hoja húmeda (RHOHU) y seca (RHOSE), relación RHOSE / RHOHU (RESEHU), porcentaje de humedad en las hojas (PHUHO), relación hoja / tallo en húmedo (RHOTAHU) y en seco (RHOTASE). Se detectaron diferencias significativas $(p<0,01)$, entre genotipos sólo para RHOTAHU y RHOTASE. Los arreglos de población tuvieron efectos significativos

${ }^{1}$ Ingeniero Agrónomo - Ph.D. Fitomejoramiento. ProfesorTitular Universidad de Córdoba, Diagonal 50 No. 8 - 105 - Barrio Villa del Río - Montería (Córdoba). mespitia@ sinu.unicordoba.edu.co

2 Ingeniero Agrónomo - M.Sc. Fisiología Vegetal. Profesor Titular Universidad de Córdoba). rmontoya@sinu.unicordoba.edu.co

3 Ingeniero Agrónomo. Asistente de Investigación. Universidad de Córdoba, lilo10_6@hotmail.com.
( $<<0,05$ y 0,01) únicamente en la expresión del RHOHU, RHOSE, RHOTAHU y RHOTASE. La interacción genotipos $x$ arreglos sólo fue significativa $(p<0.05)$ para RHOSE. El arreglo de población 20x20, mostró los más altos promedios de RHOHU (4654kg ha-1) y RHOSE (1378 kg ha-1), superando en $83 \%$, aproximadamente, al arreglo testigo 40x40. El genotipo Morita 1 expresó estadísticamente su mayor RHOSE (1586kg ha-1) en el arreglo 20x20, mientras que en Morita 2 (1416kg ha-1), se presentó en el arreglo 40x20. Se sugiere considerar el uso de los arreglos 20x20 y 40x20 en la siembra de Morita 1 y Morita 2, respectivamente, para aumentar el rendimiento de estevia en el Sinú Medio.

Palabras clave: Estevia, genotipos, rendimiento de hojas, distancias de siembra.

\section{SUMMARY}

The study objective was to evaluate the effect of three population arrangements on leaf yield in two genotypes of Stevia rebaudina Bert., under environmental conditions of Middle Sinú (Montería - Colombia), during 16 weeks of the first term of 2006. Six treatments were evaluated in a factorial arrangement $(2 \times 3)$, originating from combination of two stevia genotypes (Morita 1 and Morita 2) and three populations arrangements (20x20, 40x20 and 40x40cm between rows and plants, respectively). A completely randomized block design with four replications was used. The traits: wet leaf yield (RHOHU), dry leaf yield (RHOSE), RHOSE/RHOHU ratio (RESEHU), moisture in leaves (PHUHO), leaf/stem wet 
ratio (RHOTAHU) and leaf/stem dry ratio (RHOTASE), were analyzed. Significant differences were detected between genotypes $(p<0.01)$ only for RHOTAHU and RHOTASE. The populations arrangement had significant effects $(p<0.05$ and $p<0.01)$ in the expression of RHOHU, RHOSE, RHOTAHU and RHOTASE. The genotypes by arrangement interactions were only significant $(p<0.05)$ for RHOSE. The plant arrangement 20x20, showed the highest average RHOHU $(4654 \mathrm{~kg}$ $\left.\mathrm{ha}^{-1}\right)$ and RHOSE (1378 $\left.\mathrm{kg} \mathrm{ha}^{-1}\right)$, surpassing by about $83 \%$ the $40 \times 40$ arrangement, considered the control. Morita 1 expressed statistically the most RHOSE (1586kg ha-1) in arrangement 20x20, whereas Morita 2 (1416kg ha-1) did so at the arrangement 40x20. The use of plant arrangements 20x20 y 40x20 for Morita 1 y Morita 2 is suggested to increase yield of stevia in Middle Sinú.

Key words: Stevia, genotypes, leaves yield, planting distances.

\section{INTRODUCCIÓN}

La estevia o hierba dulce es una planta herbácea perenne perteneciente a la familia Asteraceae, cuyas hojas son fuente de glicósidos de diterpeno (esteviosidos y rebaudosidos), los cuales, se estiman tienen una capacidad endulzante 200 a 300 veces mayor que la sacarosa, extraída de la caña de azúcar, distinguiéndose de los edulcorantes artificiales por no tener sabor metálico y no ser cancerígeno (Tanaka, 1982; Soto E Del Val, 2002). Esta característica la hace una especie con gran potencial económico como endulzante de bajo poder calórico y grandes beneficios para la salud. Adicionalmente, posee alta demanda internacional por parte de Japón, China, Corea, Taiwán, Israel, Paraguay, Uruguay y Brasil (Álvarez, 2004).

Recientemente, la oficina de Administración de Alimentos y Drogas de USA (Food and Drug Administration, FDA) ha clasificado al rebaudiosido A purificado de St. rebaudiana, como endulzante, "Generalmente Reconocido como Seguro" (Generally Recognized as Safe, GRAS) en el uso de una variedad de alimentos y de bebidas, con determinadas condiciones de calidad, en niveles que oscilan entre 90 a 500mg de rebaudiosido por kilogramo de peso (FDA, 2008), lo cual, augura un futuro promisorio y sostenible para la producción, la transformación, el uso y la comercialización internacional de la estevia en Colombia, ya que el producto nacional satisface los requisitos de calidad, a nivel de hoja húmeda, seca y procesada, que exige el mercado interno y externo (Espitia et al. 2008).

Entre los principales países productores de estevia sobresalen Japón, China, Taiwan, Tailandia, Korea, Brasil, Malasia y Paraguay. Los mayores importadores, mundialmente son Japón, China, Corea, Canadá, Australia, Unión Europea y USA (Hale, 2001; Marín, 2004; MINCOPAR, 2006). El mercado total de edulcorantes de alto poder y bajo contenido calórico es equivalente entre 12,000 a 15,000t de esteviósido por año. La conquista de una pequeña fracción de este volumen, por el esteviósido, representaría cifras significativas (IIAVH, 2006).

Marín (2004) señaló que en el 2007, se tendría un consumo estimado de $100 \mathrm{t}$ de extracto de estevia y, a partir de este año, con base en un crecimiento anual del $15 \%$, para el 2015 , se esperaría un consumo de 306t, equivalentes a 9000t de azúcar, dado el poder edulcorante de los esteviósidos y rebaudiósidos. Similarmente, con base en el rendimiento en la extracción industrial de estevia del $20 \%$ y el rendimiento de 7,5t de hoja/año, para satisfacer la demanda interna del país para el 2015, se tendrían que sembrar por lo menos 200ha. Sin embargo, aún cuando no existen cifras oficiales en Colombia, extraoficialmente, se habla de un área sembrada de, aproximadamente, 100ha, lo cual, sugiere una demanda interna en aumento, sin tener en cuenta los requerimientos del mercado para exportación, que cada vez se prevén están aumentando (Espitia et al. 2008).

Las labores que demanda el cultivo, lo convierten en un sistema altamente generador de empleos rurales y se constituye como una opción para los pequeños productores (Espitia et al. 2008; Jarma, 2003). En Colombia, trabajos adelantados en la región caribe, demostraron su adaptación a la oferta ambiental de esta parte del país, convirtiéndola en una alternativa de producción económica y sostenible para los sectores agrícola y agroindustrial (Espitia et al. 2008; Jarma et al. 2006; Jarma et al. 2005; Jarma, 2003).

En el ámbito internacional, se reportan densidades de población entre 40.000 a 250.000 plantas ha ${ }^{-1}$, con arreglos en surcos sencillos dobles o triples. Las distancias de siembra más utilizadas en surcos sencillos 
son de $20 \times 20,30 \times 30,40 \times 20,45 \times 65,50 \times 20$, 50 × 50, 70 x 20 y 80 x $10 \mathrm{~cm}$ entre surcos y plantas, respectivamente (Sakaguchi $\mathcal{E}$ Tatsuiko, 1982; Brandle E Rosa, 1992; Pinaya, 1996; Lima et al. 1997; Midmore E Rank, 2002; Casaccia \& Álvarez, 2006; Zubiate, 2007; INCAGRO, 2008).

En la región Caribe, la Universidad de Córdoba ha venido desarrollando durante los últimos cinco años, trabajos de investigación en esta especie, en aspectos tecnológicos, relacionados con respuesta a la radiación (Jarma et al. 2005), multiplicación y propagación in vitro (Suárez et al. 2006; 2008), uso como edulcorante, manejo de propágulos, reconocimiento de enfermedades (Jarma, 2003), crecimiento y desarrollo (Jarma et al. 2006); sin embargo, en todos los estudios, se utilizó un solo arreglo de población de 40 x $40 \mathrm{~cm}$, entre surcos y plantas, respectivamente, lo cual, no ha permitido explorar el máximo potencial fisiológico de la especie en la región.

Los estudios de arreglos poblacionales son importantes y necesarios en las diferentes zonas productoras, porque: a) se busca que la planta maximice la expresión de su potencial genético y aproveche de manera más eficiente los factores de producción de clima y suelo, disponibles en cada localidad; b) el rendimiento depende del genotipo, ambiente y la interacción del genotipo con el ambiente; c) existe efecto significativo sobre la competencia intraplantas y con malezas, sanidad del cultivo y el aprovechamiento de la luz, agua y nutrientes; d) el rendimiento se incrementa con la densidad hasta alcanzar el óptimo, después del cual permanece constante o decrece al aumentar el número de plantas (Rodríguez, 2001; Espitia et al. 2008). Lo anterior, es de mayor interés, si se tiene en cuenta que en muchos cultivos incluidos la estevia, los agricultores establecen arreglos de poblaciones en sus siembras comerciales, sin tener en cuenta la plasticidad fenotípica de los genotipos sembrados (Rodríguez, 2001).

Diversas investigaciones han demostrado el efecto, las ventajas y las desventajas de los arreglos de población en diferentes especies, como lechuga (Lino, 2007); berenjena (Pérez et al. 2006); tomate (Barraza et al. 2004); melón (Nerson, 2002) y caraota (Díaz et al. 2001); sin embargo, las evaluaciones experimentales de distancias de siembra en estevia son escasos (Pinaya, 1996; Sakaguchi \& Tatsuiko, 1982; Sumida, 1980), aunque, existen varios reportes con recomendaciones de distancias y de densidades de siembras para el cultivo (Lima et al. 1997; Midmore \& Rank, 2002; Casaccia \& Álvarez, 2006; Zubiate, 2007; INCAGRO, 2008).

Teniendo en cuenta la ausencia de información autóctona para el manejo agronómico exitoso de la estevia, en el Valle Medio del Sinú, se consideró necesario adelantar el presente estudio, con el objetivo de evaluar el efecto de tres arreglos de población sobre el rendimiento de hoja en dos genotipos de St. rebaudina, Morita 1 y Morita 2 , variedades introducidos a esta región desde Japón, hace aproximadamente diez años y los únicos materiales que se han venido sembrando, bajo las condiciones del Sinú Medio.

\section{MATERIALES Y MÉTODOS}

El estudio, se realizó en condiciones de campo en el primer semestre de 2006, en lotes experimentales de la Universidad de Córdoba (Montería, Colombia), ubicada en las coordenadas geográficas $8^{0} 52^{\prime}$ latitud norte y $76^{\circ}$ 58' longitud Oeste, con respecto al meridiano de Greenwich y a $15 \mathrm{msnm}$. El área pertenece a la zona climática cálido-moderada, a la formación Bosque Seco Tropical (BS-T) y a la zona agroecológica $\mathrm{C}_{\mathrm{j}}$ (Palencia et al. 2006; Holdrige, 1986; IGAC, 1985). Los principales factores climáticos bajo las cuales se realizó el estudio fueron: temperatura media de 25 a $29^{\circ} \mathrm{C}$, humedad relativa de 76 a $92 \%$, brillo solar de 1,2 a 9,9 horas día ${ }^{-1}$ y precipitación de 0,0 a 40,5mm día ${ }^{-1}$ (Estación Meteorológica, Universidad de Córdoba).

Las plántulas para la siembra, se obtuvieron de ramas de plantas madres adultas (mayores de seis meses de edad) de St. rebaudiana variedades Morita 1 (M1) y Morita 2 (M2). Para evitar la floración, se realizaron podas periódicas, manteniéndose en estado vegetativo bajo condiciones de casa-malla con polisombra del $50 \%$, permanente control de plagas, de enfermedades, de fertilización y de suministro de riego. El proceso de enraizamiento, se realizó aplicando en la base de las estacas ácido naftalenacético en polvo al 0,40\%. Luego, las estacas fueron colocadas en bandejas plásticas durante un mes, empleando como sustrato la mezcla de arena y de limo en proporciones 2:1, respectivamente, desinfectada con agua caliente $\left(100^{\circ} \mathrm{C}\right)$, previamente. Se usaron bandejas plásticas por su fácil manejo, mayor eficiencia y menores costos. Al momento del transplante al sitio definitivo, las raíces de las plántulas fueron 
desinfectadas sumergiéndolas en una solución de $2 \mathrm{~g}$ $\mathrm{L}^{-1}$ de Mancozeb del $80 \%$.

En campo, se evaluaron seis tratamientos en arreglo factorial $(2 \times 3)$, originado de la combinación de los dos genotipos, M1 y M2 y tres arreglos de población (20 x 20, 40 x 20 y 40 x $40 \mathrm{~cm}$, entre surcos y plantas, respectivamente), lo cual, origina densidades de población de 250.000, 125.000 y 62.500 plantas ha ${ }^{-1}$.
Se utilizó un diseño de bloques completos al azar con cuatro repeticiones. En razón a los tres arreglos poblacionales utilizados y con el objeto de asegurar un número suficiente de plantas en competencia completa durante las 16 semanas de estudio, se emplearon tamaños de parcelas de $8,4 \mathrm{~m}^{2}$ (20 x 20), 16,8m² (40 x 20) y de $36,8 \mathrm{~m}^{2}$ ( $40 \times 40$, considerado como testigo), lo cual originó una densidad de población por unidad experimental de 210 a 230 plantas/parcela (Tabla 1).

Tabla 1. Tratamientos estudiados para la evaluación de las variables asociadas con el rendimiento en Stevia rebaudiana para Morita 1 (M1) y Morita 2 (M2), en condiciones del Sinú Medio.

\begin{tabular}{|c|c|c|c|c|c|}
\hline Tratamientos & Genotipos & $\begin{array}{c}\text { Arreglos poblacionales } \\
(\mathbf{c m})\end{array}$ & $\begin{array}{c}\text { Área / parcela } \\
\left(\mathbf{m}^{\mathbf{2}}\right)\end{array}$ & $\begin{array}{c}\text { Plantas / parcela } \\
(\#)\end{array}$ & $\begin{array}{c}\text { Plantas ha } \\
(\#)\end{array}$ \\
\hline 1 & M1 & $20 \times 20$ & $(4,2 \times 2,0)=8,4$ & 210 & 250.000 \\
\hline 2 & M1 & $40 \times 20$ & $(8,4 \times 2,0)=16,8$ & 210 & 125.000 \\
\hline 3 & M1 & $40 \times 40$ & $(18,4 \times 2,0)=36,8 f$ & 230 & 62.500 \\
\hline 4 & M2 & $20 \times 20$ & $(4,2 \times 2,0)=8,4$ & 210 & 250.000 \\
\hline 5 & M2 & $40 \times 20$ & $(8,4 \times 2,0)=16,8$ & 210 & 125.000 \\
\hline 6 & M2 & $40 \times 40$ & $(18,4 \times 2,0)=36,8$ & 230 & 62.500 \\
\hline
\end{tabular}

Se evaluaron seis variables dependientes, a saber: 1) rendimiento de hoja húmeda $(\mathrm{RHOHU}) ; 2$ ) rendimiento de hoja seca (RHOSE); 3) relación RHOSE/RHOHU (RESEHU); 4) porcentaje de humedad en las hojas (PHUHO); 5) relación hoja/tallo en húmedo (RHOTAHU) y 6) relación hoja/tallo en seco (RHOTASE). En cada unidad experimental, con el fin de estimar el RHOHU, se marcó una parcela productiva de 24 plantas, las cuales, se cosechaban a $10 \mathrm{~cm}$ de altura, mediante podas periódicas (hojas + tallos), cada vez que se iniciaba la floración en el $50 \%$ de las plantas, ello, en razón a que las hojas son el producto de interés y es en este momento fisiológico, donde la hoja acumula la mayor concentración de esteviosidos y rebaudosidos (Tanaka, 1982; Soto E Del Val, 2002). Las producciones parciales en fresco de cada unidad experimental, se empacaron en bolsas de papel, previamente identificadas, luego, se llevaron al laboratorio, en donde se procedió a separar y pesar las hojas y los tallos; los datos parciales de hojas de cada unidad experimental, se sumaron al final y se expresaron en $\mathrm{kg} \mathrm{ha}^{-1}$, teniendo en cuenta el área de cada parcela productiva.
Para obtener el RHOSE, se siguió la metodología descrita para $\mathrm{RHOHU}$ y las producciones parciales en fresco de hojas de cada unidad experimental, se secaron en horno por un periodo de 72 horas, a una temperatura de $70^{\circ} \mathrm{C}$ y se expresaron en $\mathrm{kg} \mathrm{ha}^{-1}$. La variable RESEHU, se obtuvo mediante la siguiente ecuación: RESEHU = (RHOSE/RHOHU)*100; el porcentaje de humedad en las hojas, se estimó a través de la siguiente formula: PHUHO $=[(\mathrm{RHOHU}-\mathrm{RHOSE}) / \mathrm{RHOHU}]^{*} 100$; las relaciones RHOTAHU y RHOTASE, se calcularon a través de las ecuaciones: RHOTAHU = (peso fresco de hojas/ peso fresco total de hojas más tallos)*100 y RHOTASE $=$ (peso seco de hojas/peso seco total de hojas más tallos)*100. Con los datos finales obtenidos para cada variable, se llevaron a cabo los análisis de varianza y pruebas de comparación de medias, con el uso del programa computacional GENES versión Windows (2004.2.1), desarrollado por Cruz (2004). 


\section{RESULTADOS Y DISCUSIÓN}

El análisis de varianza para las seis variables en estudio (Tabla 2) detectó diferencias estadísticas $(p<0,01)$ entre genotipos sólo para la RHOTAHU y la RHOTASE. Esto se debe a una ventaja genética de $M 2$, que expresó una mayor proporción de hoja en relación al peso total de hojas más tallos, tanto en fresco como en seco. Ello, posiblemente, se explique por la mayor área foliar, tasa absoluta de crecimiento y tasa de asimilación neta, lo cual, se traduce en mayor fotosíntesis y eficiencia en la producción diaria de biomasa por cada centímetro cuadrado de superficie foliar (Jarma et al. 2006).

Tabla 2. Cuadrados medios del análisis de varianza para las seis variables asociadas con el rendimiento ${ }^{1}$ de hojas, en dos genotipos estevia y tres arreglos de población.

\begin{tabular}{|c|c|c|c|c|c|c|c|}
\hline F. V. & G.L. & $\begin{array}{l}\text { RHOHU } \\
\left(\mathrm{kg} \mathrm{ha}^{-1}\right)\end{array}$ & $\begin{array}{l}\text { RHOSE } \\
\left(\mathrm{kg} \mathrm{ha}^{-1}\right)\end{array}$ & RESEHU (\%) & PHUHO (\%) & $\begin{array}{l}\text { RHOTAHU } \\
(\%)\end{array}$ & $\begin{array}{l}\text { RHOTASE } \\
(\%)\end{array}$ \\
\hline Bloques & 3 & $812298,92 \mathrm{~ns}$ & $134480,04 \mathrm{~ns}$ & $67,12 \mathrm{~ns}$ & $67,12 \mathrm{~ns}$ & $60,88^{*}$ & $49,05 n s$ \\
\hline Genotipos (G) & 1 & $279523,59 \mathrm{~ns}$ & $1777,44 \mathrm{~ns}$ & $1,78 \mathrm{~ns}$ & $1,78 \mathrm{~ns}$ & $7548,72^{* *}$ & $5988,83^{* *}$ \\
\hline Arreglos (A) & 2 & $9299331,01^{*}$ & $824791,96^{* *}$ & $0,58 \mathrm{~ns}$ & $0,58 \mathrm{~ns}$ & $64,87^{*}$ & $95,16^{*}$ \\
\hline$G \times A$ & 2 & $2078298,45 \mathrm{~ns}$ & $358736,81^{*}$ & $69,42 \mathrm{~ns}$ & $69,42 n s$ & $1,52 \mathrm{~ns}$ & $32,27 n s$ \\
\hline Error & 15 & 1553578,17 & 72874,22 & 43,57 & 43,57 & 15,94 & 22,06 \\
\hline Total & 23 & & & & & & \\
\hline C.V (\%) & & 33,52 & 24,28 & 21,48 & 9,52 & 6,66 & 7,44 \\
\hline Promedio & & 3717,8 & 1111,8 & 30,7 & 69,3 & 59,9 & 63,0 \\
\hline
\end{tabular}

${ }^{*},{ }^{* *}$ significativos al $5 \%$ y $1 \%$ de probabilidad, respectivamente. ns: no significativos.

1. rendimiento de hoja húmeda (RHOHU), rendimiento de hoja seca (RHOSE), relación RHOSE/RHOHU (RESEHU), porcentaje de humedad en las hojas (PHUHO), relación hoja/tallo en húmedo (RHOTAHU) y relación hoja/tallo en seco (RHOTASE).

En la fuente de variación arreglos de población, se detectaron diferencias significativas al $1 \%$ de probabilidad para RHOSE y al 5\% de probabilidad RHOHU, RHOTAHU y RHOTASE. Ello señala, que al menos uno de los arreglos utilizados, tuvo un efecto significativo en la expresión promedio de tales variables; sin embargo, ese efecto fue diferente en uno de los genotipos para el RHOSE, ya que la interacción genotipo por arreglos (G $x A)$, sólo fue significativo $(p<0,05)$ para esta variable. La ausencia de diferencias significativas en la interacción $G \times$ A para el resto de las variables, sugiere que los dos genotipos, a pesar de tener diferencias fisiológicas en floración (M1 =50 y M2=90 días después del transplante), mostraron un comportamiento estadísticamente similar en los tres arreglos de población estudiados, lo que señala que la respuesta agronómica de los dos genotipos en RHOHU, RESEHU, PHUHO, RHOTAHU y RHOTASE, no se vio afectada por los microambientes generados por las tres densidades de población utilizadas y, por lo tanto, existe un único genotipo superior en los tres arreglos de población; resultados similares han sido encontrados para el rendimiento de hoja en fresco de estevia en Bolivia (Pinaya, 1996).

Rendimiento de hoja húmeda (RHOHU) y seca (RHOSE): Los promedios entre genotipos para $\mathrm{RHOHU}$ y RHOSE (Tabla 3) corroboran la ausencia de diferencias significativas detectadas en el análisis de varianza para estas dos variables e indican, además, que los dos genotipos mostraron un rendimiento de hoja en fresco y seco, estadísticamente, similar. El rendimiento acumulado de hoja fresca durante las 16 semanas del estudio, osciló entre 3610 a 3826 kg ha-1, para M2 y M1, respectivamente, con una media general de $3718 \mathrm{~kg}$ ha $^{-1}$, aproximadamente. A su vez, el rendimiento de hoja seca osciló entre 1103 (M2) y $1120 \mathrm{~kg} \mathrm{ha}^{-1}$ (M1), con una media general de $1112 \mathrm{~kg} \mathrm{ha}^{-1}$. Rendimientos de hoja seca ligeramente inferiores $\left(967 \mathrm{~kg} \mathrm{ha}^{-1}\right)$ son 
Tabla 3. Promedios por genotipo para las seis variables asociadas con el rendimiento'1.

\begin{tabular}{|c|c|c|c|c|c|c|}
\hline GENOTIPO & $\begin{array}{c}\text { RHOHU } \\
\left.\mathbf{( k g ~ h a}^{-1}\right)\end{array}$ & $\begin{array}{c}\text { RHOSE } \\
\left.\mathbf{( k g ~ h a}^{-1}\right)\end{array}$ & RESEHU (\%) & PHUHO (\%) & RHOTAHU (\%) & RHOTASE (\%) \\
\hline M1 & $3825,8 \mathrm{a}$ & $1120,4 \mathrm{a}$ & $30,4 \mathrm{a}$ & $69,5 \mathrm{a}$ & $42,2 \mathrm{~b}$ & $47,2 \mathrm{~b}$ \\
\hline M2 & $3609,9 \mathrm{a}$ & $1103,1 \mathrm{a}$ & $30,9 \mathrm{a}$ & $69,0 \mathrm{a}$ & $77,6 \mathrm{a}$ & $78,8 \mathrm{a}$ \\
\hline
\end{tabular}

Promedios con la misma letra no son estadísticamente diferentes, según la prueba de Duncan.

1. Rendimiento de hoja húmeda (RHOHU), rendimiento de hoja seca (RHOSE), relación RHOSE/RHOHU (RESEHU), porcentaje de humedad en las hojas (PHUHO), relación hoja/tallo en húmedo (RHOTAHU) y relación hoja/tallo en seco (RHOTASE).

reportados en las condiciones del Valle del Cauca, por Bonilla et al. (2007), para las dos primeras cosechas, utilizando distancias de 40 x $40 \mathrm{~cm}$. Rendimientos de hoja fresca inferiores son reportados por Brandle $\mathcal{E}$ Rosa (1992), quienes en condiciones de campo en Canadá obtuvieron 2144 a $3494 \mathrm{~kg} \mathrm{ha}^{-1}$, utilizando arreglos de siembra de 30 x 30cm, mientras que Lima et al. (1997), en Brasil, con distancias de 50 × 50 y 50 × $20 \mathrm{~cm}$, reportan rendimientos de $2300 \mathrm{~kg} \mathrm{ha}^{-1}$, bajo condiciones de plantación comercial.

La Tabla 4 señala que las distancias de siembra de $20 \mathrm{x}$ 20 y 40 x $20 \mathrm{~cm}$ entre surcos y plantas, respectivamente, fueron los arreglos de población, en donde los genotipos expresaron, estadísticamente, los mayores rendimientos, tanto de hoja fresca (4654 y 3960kg ha-1) como seca (1379 y $\left.1201 \mathrm{~kg} \mathrm{ha}^{-1}\right)$, superando a la distancia comercial utilizada en Córdoba, de 40 x $40 \mathrm{~cm}$, en $83 \%$ a $56 \%$ en el rendimiento de hoja fresca, y en $82 \%$ a $59 \%$ en el rendimiento de hoja seca, respectivamente. Esta respuesta, se debe, como lo señalan algunos autores (Rodríguez, 2001; Espitia et al. 2008), al mayor número de plantas por unidad de área (mayor densidad de población) y la arquitectura de planta arbustiva, que le permiten mejor aprovechamiento de la radiación solar (mayor captación neta de luz por parte del cultivo, aunque por planta puede ser menor, por mayor competencia intraplantas) agua y nutrientes, aun siendo considerada una planta C3 (Midmore E Rank, 2002), posiblemente, a la menor incidencia de malezas, lo cual, a su vez, facilita una mayor producción de biomasa con respecto a la distancia de 40 x 40.

La ventaja que ofrecen los arreglos de siembra con mayor densidad de población radica en que es posible aumentar el rendimiento de hoja fresca y seca en estevia en el Sinú Medio, con el uso de menores distancias de siembra entre surcos y plantas. Resultados similares son

Tabla 4. Promedios por arreglos de población para las seis variables asociadas con el rendimiento.

\begin{tabular}{|c|c|c|c|c|c|c|}
\hline $\begin{array}{c}\text { ARREGLOS } \\
(\mathbf{c m})\end{array}$ & $\begin{array}{c}\text { RHOHU } \\
\left(\mathbf{k g ~ h a}^{-1}\right)\end{array}$ & $\begin{array}{c}\text { RHOSE } \\
\left(\mathbf{k g ~ h a}^{-1}\right)\end{array}$ & RESEHU (\%) & PHUHO (\%) & RHOTAHU (\%) & RHOTASE (\%) \\
\hline$(20 \times 20)$ & $4654,4 \mathrm{a}$ & $1378,5 \mathrm{a}$ & $30,9 \mathrm{a}$ & $69,1 \mathrm{a}$ & $63,2 \mathrm{a}$ & $65,6 \mathrm{a}$ \\
\hline$(40 \times 20)$ & $3959,9 \mathrm{a}$ & $1201,4 \mathrm{a}$ & $30,4 \mathrm{a}$ & $69,6 \mathrm{a}$ & $57,8 \mathrm{~b}$ & $59,1 \mathrm{~b}$ \\
\hline$(40 \times 40)$ & $2539,2 \mathrm{~b}$ & $755,4 \mathrm{~b}$ & $30,8 \mathrm{a}$ & $69,2 \mathrm{a}$ & $58,8 \mathrm{~b}$ & $64,5 \mathrm{a}$ \\
\hline
\end{tabular}

Promedios con la misma letra no son estadísticamente diferentes, según la prueba de Duncan.

1. Rendimiento de hoja húmeda (RHOHU), rendimiento de hoja seca (RHOSE), relación RHOSE/RHOHU (RESEHU), porcentaje de humedad en las hojas (PHUHO), relación hoja/tallo en húmedo (RHOTAHU) y relación hoja/tallo en seco (RHOTASE). 
reportados en otros países por varios autores (Pinaya, 1996; Sakaguchi E Tatsuiko, 1982; Sumida, 1980; Midmore E Rank, 2002; Casaccia E Álvarez, 2006; Zubiate, 2007; INCAGRO, 2008), lo cual, los ha llevado a recomendar densidades de población de hasta 250 mil plantas ha ${ }^{-1}$.

En la tabla 5, se muestra el RHOSE, bajo los tres arreglos de población estudiados. Se observa claramente que el genotipo M1 expresó su mayor potencial genético en rendimiento de hoja seca $\left(1586 \mathrm{~kg} \mathrm{ha}^{-1}\right)$, cuando se sembró a una distancia de 20 x $20 \mathrm{~cm}$ entre surcos y plantas, respectivamente, mientras que $M 2$, lo hizo en el arreglo 40 x 20 y 20 x $20 \mathrm{~cm}$ (1416 y $\left.1171 \mathrm{~kg} \mathrm{ha}^{-1}\right)$. Ello sugiere, como lo reporta Jarma et al. (2006), que los dos genotipos estudiados por sus diferencias en precocidad a floración (45 días), altura de planta $(25 \mathrm{~cm})$, área foliar $\left(0,50 \mathrm{~cm}^{2}\right)$, tasa absoluta de crecimiento y tasa de asimilación neta, se ven afectados de forma diferencial en su respuesta en RHOSE y, a su vez, explica la interacción significativa $(p<0,05)$ genotipo $x$ arreglo detectada en el análisis de varianza (Tabla 2). Los rendimientos de hoja seca encontrados en este estudio son superiores a los reportados en M1 y M2 por algunos productores en el departamento de Córdoba (1000kg ha-1) y a los obtenidos en las condiciones del Valle del Cauca por Bonilla et al. (2007), quienes utilizando distancias de $40 \mathrm{x} 40 \mathrm{~cm}$ obtuvieron rendimientos promedios de $967 \mathrm{~kg} \mathrm{ha}^{-1}$ para las dos primeras cosechas. Esta diferencia, se explica posiblemente, no sólo por las distancias de siembra, sino también por la aplicación de mejores prácticas agronómicas en el ensayo, en cuanto a riego, fertilización, control de malezas, insectos plagas y enfermedades, lo cual, a su vez, permitió que los dos genotipos estudiados expresaran su mayor potencial genético en altura de planta $(M 1=39,3 \mathrm{~cm}$ y $M 2=64,8 \mathrm{~cm})$ y área foliar $\left(M 1=1,94 \mathrm{~cm}^{2}\right.$ y $M 2=2,33$ $\left.\mathrm{cm}^{2}\right)$.

Porcentaje de humedad en las hojas (PHUHO) y relación entre el rendimiento de hoja seca y húmeda (RESEHU): El comportamiento promedio de los dos genotipos de estevia considerados en el estudio para estas dos variables (Tabla 3), señala que M1 y M2 presentaron similar $\mathrm{PHUHO}$, con promedios que oscilaron entre 69,5\% (M1) y 69,0\% (M2). Los anteriores promedios indican que por cada $100 \mathrm{~kg}$ de hoja fresca que se cosechan en estos genotipos de estevia, $69 \mathrm{~kg}$ aproximadamente, corresponden al agua contenida en las hojas (humedad).

Los dos genotipos fueron igual de eficientes en su proceso de conversión del rendimiento de hoja húmeda a hoja seca, que se mide a través de la variable RESEHU, ya que sus promedios no fueron estadísticamente diferentes, presentando valores entre 30,4\% (M1) y 30,9\% (M2), valores que sugieren que por cada $100 \mathrm{~kg}$ de hoja fresca que se producen en cada genotipo, se obtienen en promedio $31 \mathrm{~kg}$, aproximadamente, de hoja seca. La capacidad, estadísticamente igual, de conversión de hoja húmeda a hoja seca en los dos genotipos, se explica porque ambos presentan similares rendimientos y contenidos de humedad en las hojas.

La relación de conversión aproximada 3:1 de hoja húmeda a seca obtenida es de mucho interés a nivel comercial para productores, comercializadores y procesadores, ya que facilita determinar un precio

Tabla 5. Rendimiento promedio de hoja seca, RHOSE ( $\left.\mathrm{kg} \mathrm{ha}^{-1}\right)$ para Morita 1 y Morita 2, bajo tres arreglos de población (20x20, 40x20 y $40 \times 40 \mathrm{~cm})$.

\begin{tabular}{|l|c|c|c|c|}
\hline \multirow{2}{*}{ GENOTIPOS } & \multicolumn{3}{|c|}{ ARREGLOS POBLACIONALES (cm) } & \multirow{2}{*}{ PROMEDIO } \\
\cline { 2 - 4 } & $\mathbf{2 0 \times 2 0}$ & $\mathbf{4 0 \times 2 0}$ & $\mathbf{4 0 \times 4 0}$ & \\
\hline Morita 1 & $1586 \mathrm{a}$ & $987 \mathrm{bc}$ & $788 \mathrm{bc}$ & 1120 \\
\hline Morita 2 & $1171 \mathrm{ab}$ & $1416 \mathrm{a}$ & $722 \mathrm{c}$ & 1103 \\
\hline PROMEDIO & 1379 & 1202 & 755 & 1112 \\
\hline
\end{tabular}

Promedios con la misma letra no son estadísticamente diferentes, según la prueba de Duncan. 
justo, cuando se vende la producción de hoja en seco y no fresca. Esto permite una producción con mayor valor agregado, con secamiento de las hojas al sol, como se realiza en Córdoba. Resultados similares han sido reportados por Brandle E Rosa (1992), Lima et al. (1997) y Jarma (2003), con valores de RESEHU, que han oscilado entre 30 y 33\%, con secamiento de hojas en estufas de secado (Brandle E Rosa, 1992; Lima et al. 1997) y al sol (Jarma, 2003).

En la tabla 4, se corrobora la ausencia de efecto significativo de las distancias de siembra sobre las variables PHUHO y RESEHU detectados en el análisis de varianza (Tabla 2); los tres arreglos muestran promedios no significativos en cada variable, con valores que oscilan alrededor de $69,3 \%$ para el $\mathrm{PHUHO}$ y $30,7 \%$ para la RESEHU. Estos resultados, se deben, posiblemente, y como lo mencionan algunos autores (Jarma, 2005; Pinaya, 1996; Brandle E Rosa, 1992; Sakaguchi $E$ Tatsuiko, 1982; Sumida, 1980) a que las dos características son muy poco afectadas por las variaciones ambientales, son controladas por uno o pocos genes mayores y los dos genotipos difieren muy poco genéticamente para tales caracteres. A pesar de lo anterior, es posible detectar efectos ambientales en la estimación del PHUHO y RESEHU, cuando se realizan muestreos en diferentes horas del día o épocas de siembra (Pinaya, 1996; Brandle \& Rosa, 1992).

Relación hoja/tallo en húmedo (RHOTAHU) y seco (RHOTASE): La prueba de comparación de medias (Tabla 3) señala que las diferencias significativas $(p<0,01)$ entre los genotipos (Tabla 2), estuvieron explicada por el genotipo $M 2$, el cual, con valores promedios de $77,6 \%$ y $78,8 \%$ en su RHOTAHU y su RHOTASE respectivamente, superó a $M 1$ en un 35,4 y 31,6 puntos porcentuales en la RHOTAHU y RHOTASE, respectivamente. Lo anterior, se debe, como lo señala Jarma et al. (2006; 2005), a que las hojas de M2 presentan un mayor tamaño, que le permite responder de manera más eficiente a la máxima radiación incidente y lograr mayor fijación de $\mathrm{CO}_{2}$, lo que, se traduce en mayor acumulación de área foliar y ganancia de biomasa. No obstante, esta mayor eficiencia fisiológica en la relación hojas/tallo de M2 con respecto a $M 1$, no se reflejó estadísticamente en el rendimiento acumulado final de hoja húmeda y seca, ya que los dos genotipos no mostraron diferencias significativas entre ellas.
La presencia de efectos estadísticos entre genotipos para la RHOTAHU y la RHOTASE, frente a la ausencia en el PHUHO y RESEHU, señala diferencias genéticas importantes entre Morita 1 y 2, para las dos primeras variables, pero no para las dos últimas. Esas diferencias genéticas, se expresan en una mejor distribución de fotosintatos hacia las hojas frente al tallo en M2 comparada con M1, lo cual, no necesariamente se tiene que reflejar en el PHUHO y RESEHU, ya que en éstas, lo que se evalúa es el contenido humedad en las hojas y no la eficiencia fisiológica de los dos genotipos.

La tabla 4 señala que la distancia de siembra de 20 x $20 \mathrm{~cm}$ entre surcos y plantas, respectivamente, fue el arreglo de población, en donde en promedio los genotipos de estevia expresarán, estadísticamente, la mayor RHOTAHU y la mayor RHOTASE, con valores de $63,2 \%$ y $65,6 \%$, respectivamente, superando a la distancia de siembra comercial utilizada en Córdoba de 40 x $40 \mathrm{~cm}$, en 4,4 puntos porcentuales en la RHOTAHU, pero sin ser estadísticamente superior a ésta en RHOTASE.

Como ya se mencionó para el rendimiento, esta respuesta diferencial se debe y como señalan algunos autores (Rodríguez, 2001; Espitia et al. 2008) a que el arreglo de siembra de $20 \times 20 \mathrm{~cm}$, le confiere el espaciamiento óptimo a las plantas de estevia para que puedan maximizar la expresión de su potencial genético en su defensa y aprovechamiento de manera más eficientemente los factores de producción de clima y de suelo disponibles en la localidad del estudio, resultado, posiblemente, por una mayor distribución de fotoasimilados a la producción de hojas, produciendo tallos más delgados y menos ramificados. Lo anterior, a su vez facilita una mayor eficiencia fisiológica en la producción de biomasa en hojas con relación a tallos.

La ventaja que ofrecen los arreglos de siembra con mayor densidad de población utilizado en el estudio señala que no sólo es posible aumentar la relación hoja/tallo (húmedo y seco), sino, además, el rendimiento de hoja fresca y seca en estevia en el Sinú Medio, con el uso de distancias de siembras de 20 x $20 \mathrm{~cm}$ entre surcos y plantas, respectivamente, ya que el costo que implica el uso de mayor cantidad de esquejes para siembra no es significativo en la estructura de los costos de producción (Espitia et al. 2008). Estos resultados concuerdan con las recomendaciones en otros países 
por varios autores (Pinaya, 1996; Sakaguchi $\&$ Tatsuiko, 1982; Sumida, 1980; Midmore E Rank, 2002; Casaccia E Álvarez, 2006; Zubiate, 2007; INCAGRO, 2008), en el sentido de aumentar los rendimientos de hojas a través del aumento de la densidad de siembra.

\section{CONCLUSIONES}

El uso de la distancia de siembra de $20 \times 20 \mathrm{~cm}$ permite aumentar la relación hoja/tallo (húmedo y seco), el rendimiento de hoja fresca y seca en estevia en el Sinú Medio, en comparación con la distancia comercial utilizada por los productores de 40 x $40 \mathrm{~cm}$.

El genotipo Morita 1 expresó su mayor rendimiento de hoja seca en el arreglo 20 x 20, mientras que en Morita 2, se presentó en el arreglo 40 x $20 \mathrm{~cm}$, por ello, se sugiere considerar el uso de estos arreglos poblacionales, para aumentar el rendimiento de estevia en el Sinú Medio.

En promedio los dos genotipos mostraron un rendimiento de hoja en fresco y seco estadísticamente similar.

\section{BIBLIOGRAFÍA}

ÁLVAREZ, J. 2004. Stevia rebaudiana Bertoni. Universidad EAFIT. Departamento de Negocios Internacionales. Documento elaborado para la Secretaría de Agricultura y Desarrollo Rural de Antioquia. Medellín. 71p.

BARRAZA, F; FISCHER, G; CARDONA, C. 2004. Estudio del proceso de crecimiento del cultivo de tomate (Lycopersicom esculentum Mill) en el Valle del Sinú Medio, Colombia. Agronomía Colombiana. 22:223-228.

BONILLA, C.R., SÁNCHEZ, M.S., PERLAZA, D.F. 2007. Evaluación de métodos de propagación, fertilización nitrogenada y fenología de estevia en condiciones del Valle del Cauca. Acta Agronómica. 56(3):131-134.

BRANDLE, J.E.; ROSA, N. 1992. Heritability for yield, leaf-stem ratio and stevioside content estimated from a landrace cultivar of Stevia rebaudiana. Can. J. Plant Sci. 72:1263-1266.

CASACCIA, J.; ÁLVAREZ, E. 2006. Recomendaciones técnicas para una producción sustentable del ka'a he'e (Stevia rebaudiana (Bertoni) en el Paraguay. Instituto Agronómico Nacional. Programa de Investigación de KA'A HE’E. 53p. Disponible desde Internet en: http://www.steviaparaguaya.com. py/recomendaciones-tecnicas_kaahee.pdf (con acceso el 25/01/2009).

CRUZ, C.D. 2004. Programa GENES. Versao Windows. Aplicativo Computacional em Genética e Estatística. Editora UFV. Universidade Federal de Viçosa. Disponible desde Internet en: http:// www.ufv.br/dbg/genes/genes.htm (con acceso el 16/01/05).

DÍAZ, C.; FIGUEROA, N.; WARNOCK, R. 2001. Estudio del crecimiento y desarrollo de la caraota (Phaseolus vulgaris L.) bajo diferentes densidades de población. Rev. Fac. Agronomía. 27(2):121126.

ESPITIA, M., MONTOYA, R., JARMA, A. 2008. Stevia en el Caribe Colombiano. Gráficas del Caribe Ltda. Montería - Córdoba. 84p.

FDA FOOD AND DRUG ADMINISTRATION. 2008. Agency Response Letter GRAS Notice No. GRN 000252. Rebaudioside A purified from Stevia rebaudiana (Bertoni). CFSAN/Office of Food Additive Safety. December 17. Disponible desde Internet en: http://www.cfsan.fda.gov/ rdb/opag253.html (con acceso el 5/01/2009).

HALE, A. 2001. Stevia rebaudiana: Propiedades, mercados y factibilidad de producción de un cultivo de venta al contado en Bolivia. USAID/ Bolivia. 21p. Disponible desde Internet en: http:// pdf.usaid.gov/pdf_docs/PNACU375.pdf (con acceso el 10/02/2009).

HOLDRIGE, L. 1986. Ecología basada en las zonas de vida. Instituto Interamericano de Ciencias Agrícolas. San José. Costa Rica. 214p.

INCAGRO. 2008. Manual técnico de producción de Stevia. Disponible desde Internet en: http://www. incagro.gob.pe/ incagro/apc-aa-files/e457b334 6514303468089b655b420d50/Manual_T_cnico_ de_Stevia.pdf (con acceso el 25/01/2009). 
IGAC INSTITUTO GEOGRÁFICO AGUSTÍN CODAZZI. 1985. Zonificación Agroecológica de Colombia. Memoria explicativa. Litografia IGAC. Bogotá, D. C. 57p.

IIAVH INSTITUTO DE INVESTIGACIONES ALEXANDER VON HUMBOLDT - BIOCOMERCIO. 2006. Investigación de mercados de Edulcorantes Naturales. 173p. Disponible desde Internet en: http://www.humboldt.org.co/obio/simbio/ documentos/ESTUDIO\%20EDULCORANTES\%2 OHUMBOLDT.pdf (con acceso el 16/01/2009).

JARMA, A. 2003. Stevia rebaudiana Bert., alternativa de sustitución de cultivos ilícitos en Colombia. Documento interno. Informe Final de Proyecto. U. de Córdoba, Fac. Ciencias Agrícolas. Montería. 267p.

JARMA, A.; RENGIFO, T.; ARAMÉNDIZ, H. 2006. Fisiología de estevia (Stevia rebaudiana Bertoni) en función de la radiación en el Caribe colombiano. II. Análisis de crecimiento. Agr. Col. 24(1):38-47.

JARMA, A.; RENGIFO, T.; ARAMÉNDIZ, H. 2005. Aspectos fisiológicos de estevia (Stevia rebaudiana Bertoni) en el Caribe colombiano: I. Efecto de la radiación incidente sobre el área foliar y la distribución de biomasa. Agr. Col. 23(2):207-216.

LIMA FILHO, O.F. de; MALAVOLTA, E; SENA, O.A. de; CARNEIRO, J.W.P. 1997. Absorção e acumulação de nutrientes em estévia Stevia rebaudiana (Bert.) Bertoni: I. Macronutrientes. Sci. Agric. 54(1-2):1422.

LINO E., L. 2007. Respuesta de tres variedades de lechuga (Lactuca sativa, L.) a diferentes densidades de plantación bajo régimen de cultivo semiprotegido. Rev. Agrotécnia de Cuba. 21:12-15.

MARÍN, W. 2004. Sondeo de Mercado de la Estevia. Instituto de Investigación de Recursos Biológicos Alexander von Humboldt. Bogotá-Colombia. 66p. Disponible desde Internet en: http://www. humboldt.org.co/obio/simbio/documentos/Sond eo\%20del\%20Mercado\%20de\%20Estevia.pdf (con acceso el 7/02/2009).
MIDMORE, D.J.; RANK, A.H. 2002. A new rural industry - Stevia - to replace imported chemical sweeteners. RIRDC Web Publication No W02/022. 50p. Disponible desde Internet en: http://www. rirdc.gov.au/reports/NPP/02-022.pdf (con acceso el 16/02/2009).

MINCOPAR MINISTERIO DE INDUSTRIA Y COMERCIO DE PARAGUAY (2006. Diagnóstico cadena stevia. Foro de competitividad de la cadena productiva de la Stevia. 42p. Disponible desde Internet en: http://www.programapymes.gov.py/descarga/ stevia/diagnosticos/diagnostico.pdf (con acceso el 10/02/2009).

NERSON, H. 2002. Relation between plant density and fruit and seed production in muskmelon. J. Am. Soc. Hort. Sci. 127(5):855-859.

PALENCIA, G.; MERCADO, T.; COMBATT, E. 2006. Estudio Agroclimático del departamento de Córdoba. Ed. Gráficas del Caribe Ltda. Montería. 126p.

PÉREZ, M.; MONTOYA, R.; CARDONA, C.; ARAMÉNDIZ, H.; ROBLES, J. 2006. Efecto de cuatro densidades de población sobre el crecimiento del fruto de berenjena (Solanum melongena L.). Rev. Temas Agrarios. 11(2):14-25.

PINAYA, R.A. 1996. Efecto de la densidad de siembra sobre el rendimiento de steviosido en cultivo de stevia (Stevia rebaudiana Bertoni). Disponible desde Internet en: http://orton.catie.ac.cr/cgi-bin/ wxis. exe/?IsisScript $=$ AGRISUM.xisEmethod $=$ pos $\mathrm{t}$ Eformato $=2 \mathcal{E}$ cantidad $=1 \mathcal{E}$ expresion $=\mathrm{mfn}=00$ 0376 (con acceso el 5/02/2009).

RODRÍGUEZ, L. 2001. Densidad de población vegetal y producción de materia seca. Rev. Comalfi (Bogotá). 27:31-38.

SAKAGUCHI, M.; TATSUIKO, K. 1982. As pesquisas japonesas com Stevia rabaudiana (Bert.) Bertoni e o esteviosideo. Ciencia e Cultura. 34(2):235-248.

SOTO, A.; DEL VAL, S. 2002. Extracción de los principios edulcorantes de la Stevia rebaudiana. Ciencias Agr. y Tecnología de los Alimentos. 20:5-9. 
SUÁREZ, I.; SALGADO, J. 2008. Propagación In Vitro de Stevia rebaudiana BERT. (AsteraceaeEupatorieae) a través de organogénesis. Rev. Temas Agrarios. 13(1):40-48.

SUÁREZ, I.; ESPITIA, M.; PERTUZ, I. 2006. Efecto de auxinas y citocininas en la multiplicación y enraizamiento In Vitro de Stevia rebaudiana Bertoni. Fitotecnia Colombiana. 6(2):1-8.

SUMIDA, T. 1980. Studies on Stevia rebaudiana Bertoni as a new possible crop for sweetning resource in Japan. J. Cent. Agric. Exp. Sta. 31:1-71.
TANAKA, O. 1982. Steviol-glycosides: New natural sweeteners. Trends in Analytic Chemistry (Netherlands). 1:246-248.

ZUBIATE, F. 2007. Manual del Cultivo de la Stevia (Yerba Dulce). Disponible desde Internet en: http://www. engormix.com/s_articles_view.asp?art $=1337$ (con acceso el 12/02/2009).

Recibido: Enero 31 de 2009

Aceptado: Abril 30 de 2009 